EchoGéo

\title{
Un état des lieux du Brésil en 2017
}

\section{Hervé Théry}

\section{(2) OpenEdition}

Journals

\section{Electronic version}

URL: https://journals.openedition.org/echogeo/15010

DOI: 10.4000/echogeo.15010

ISSN: 1963-1197

\section{Publisher}

Pôle de recherche pour l'organisation et la diffusion de l'information géographique (CNRS UMR 8586)

\section{Electronic reference}

Hervé Théry, "Un état des lieux du Brésil en 2017", EchoGéo [Online], 41 | 2017, Online since 28 September 2017, connection on 01 August 2021. URL: http://journals.openedition.org/echogeo/ 15010 ; DOI: https://doi.org/10.4000/echogeo.15010

This text was automatically generated on 1 August 2021.

EchoGéo est mis à disposition selon les termes de la licence Creative Commons Attribution - Pas d'Utilisation Commerciale - Pas de Modification 4.0 International (CC BY-NC-ND) 


\title{
Un état des lieux du Brésil en 2017
}

\author{
Hervé Théry
}

Le Brésil est tantôt vu comme promis à devenir une des grandes puissances de demain, tantôt comme un pays encore largement sous-développé, corrompu et violent. Les deux visions ont une part de vérité, car ces deux réalités - et d'autres encore - coexistent, plus ou moins bien, dans cet immense pays émergent. Le caractériser n'est pas facile car, selon Alfredo Valladão, politiste brésilien et président de l'Advisory Board de la EUBrasil Association (Bruxelles), « le problème du Brésil est que tout ce qu'on dit sur lui ... est vrai». Et comme le disait Tom Jobim ${ }^{1}$, «Le Brésil n'est pas un pays pour débutants ", entendant par là qu'il est plein de complexités, pas toujours apparentes, qui peuvent révéler des pièges pour les commentateurs trop pressés qui croient le comprendre en huit jours alors que sa réalité est plus compliquée qu'il n'y paraît à première vue. C'est d'ailleurs de longue date une spécialité brésilienne que de donner à voir aux étrangers ce qu'ils veulent voir, il existe même pour cela une expression consacrée, "para inglês ver» (" pour montrer aux Anglais »), qui remonte à un moment de tension avec la Grande-Bretagne où l'empereur du Brésil avait fait réarmer les forts de l'entrée de la paix de Rio pour montrer à la Royal Navy sa volonté de résister. Mais comme les canons n'étaient pas réellement en état de fonctionner l'expression a pris une nette connotation de tromperie ou au moins de volonté de jeter de la poudre aux yeux.

2 Pour illustrer ces ambiguïtés ${ }^{2}$, on peut recenser quelques-unes des couvertures que l'hebdomadaire britannique The Economist a récemment consacrées au Brésil (illustration 1). Il avait choisi la crise qui le frappait alors comme thème de sa première « une » de 2016, sous le titre «La chute du Brésil » et une image de la présidente Dilma Rousseff tête basse et il annonçait une « année désastreuse ». Ce n'était pas sa première couverture consacrée à ce pays et la séquence de celles-ci est révélatrice du changement de son image dans la presse internationale. Trois d'entre elles représentaient le Christ du Corcovado, qui domine la baie de Rio de Janeiro : sur la première (en novembre 2009), il décollait comme une fusée et le titre était «Le Brésil décolle ». Sur la seconde (en septembre 2013), il retombait en vrille et le titre était « Le Brésil a-t-il tout gâché ? ». Sur la troisième (en avril 2016), la statue levait des deux bras une pancarte marquée «SOS » et le titre était «La trahison du Brésil». Deux autres 
reprenaient des clichés quelque peu éculés pour attirer l'attention sur les difficultés économiques du pays, le Carnaval (sous le titre «Le bourbier brésilien », en février 2015) et la chanteuse Carmen Miranda, fameuse dans les années 1940 pour ses chapeaux ornés de fruits tropicaux (sous le titre «Pourquoi le Brésil a besoin de changements », en octobre 2014).

Illustration 1 - Couvertures consacrées au Brésil par The Economist

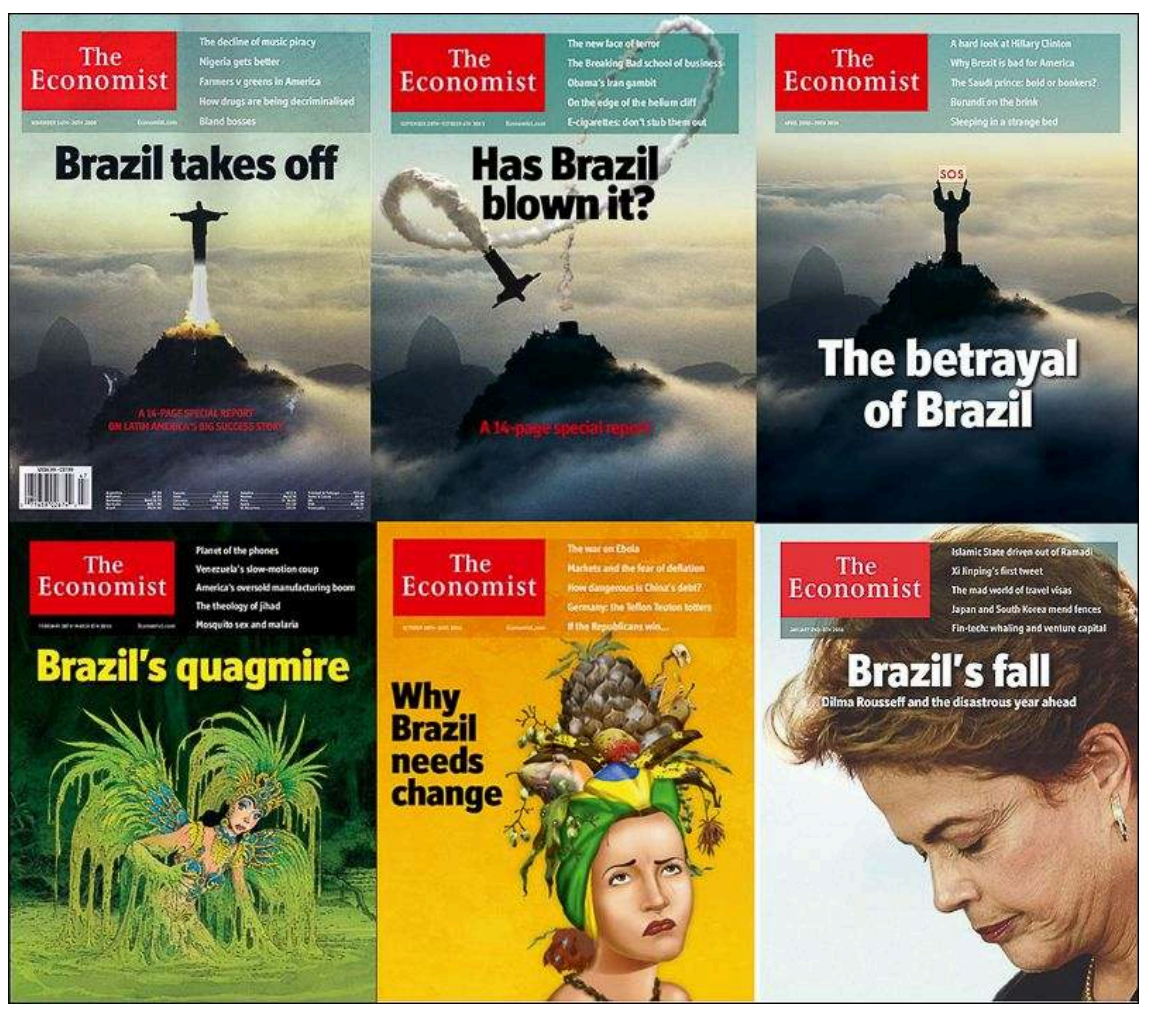

3 Pour tenter de mieux cerner cette réalité contradictoire, et la mettre en perspective avec celles d'un autre pays dit aussi émergent, ce dossier vise à reprendre, développer et prolonger sur le cas du Brésil les trois thèmes du dossier qu'Echogéo avait consacré à l'Inde, «Inde: le grand écart spatial »3, et plus particulièrement l'écart spatial, dont les relations avec le processus d'émergence restent à analyser, au Brésil comme en Inde.

4 L'appel à contribution proposait d'examiner quelques hypothèses, en précisant bien qu'il s'agissait là d'une liste non exhaustive :

- La métropolisation en cours du réseau urbain se traduit-elle pour autant par une perte de vitesse des petites villes et par la croissance concentrée dans seulement quelques grandes régions urbaines?

- Ces grands écarts existent-ils aussi à l'intérieur même des espaces ruraux ?

-Quel est l'écart entre intentions et réalités en matière d'environnement? Celui-ci est-il menacé (notamment par le déboisement de l'Amazonie), aggravant (ou non) le changement climatique, et quels sont les effets des politiques publiques mises en place pour sa protection?

- Quel est l'écart entre mythes (que ce soit Eldorado ou Enfer vert) et réalités (pionnières, urbaines, énergétiques) en Amazonie, une région dont le sort attire toujours l'attention de l'opinion publique mondiale? 
- À l'échelle continentale, l'écart s'accroît-il entre le géant brésilien et ses voisins sudaméricains?

Les articles qui nous ont été proposés ont répondu à la plupart de ces questions (sauf à la dernière, qui devra être reposée), mais aussi à bien d'autres, si bien que l'ensemble constitue un véritable état des lieux du pays en 2017. Mais avant de les présenter, groupés en fonction de ces questions initiales et d'une autre dimension apparue dans les propositions qui nous ont été faites, nous avons jugé nécessaire de présenter d'abord quelques cartes de repérage pour que les lecteurs d'Echogéo puissent mieux localiser ce dont parlent les auteurs des articles.

\section{Cartes de repérage et de mise en situation}

La première image est une carte de localisation qui permettra de situer les régions, les États et les capitales auxquels font références les différents articles présentés dans ce dossier.

Illustration 2- Régions, États et capitales

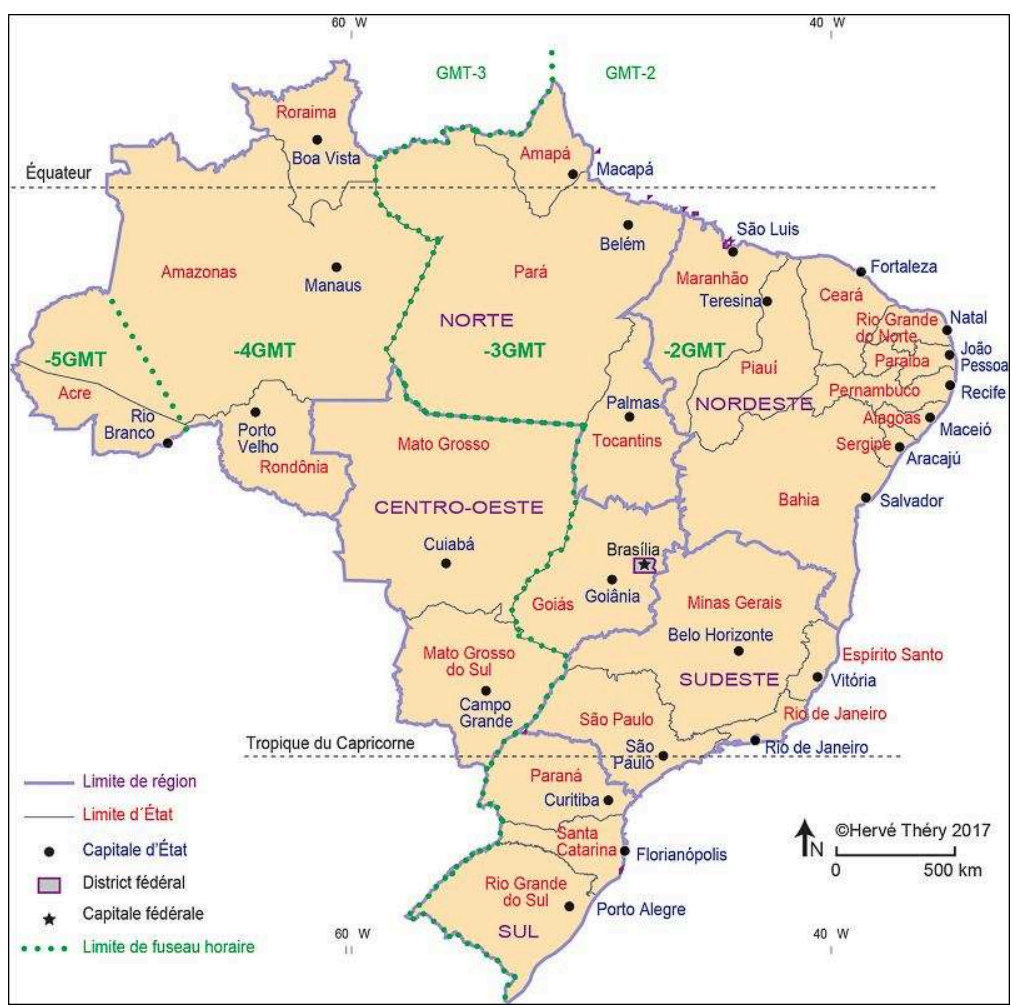

7 Ces États et ces villes sont loin d'avoir le même poids et de connaître la même évolution démographique. On peut le vérifier avec des données très récentes puisque l'IBGE (Institut brésilien de géographie et statistique) a publié le 30 août les chiffres de population par commune pour $2017^{4}$, comme il a obligation de le faire annuellement, pour ajuster - entre autres - les subventions du Fonds de participation des États et des municipalités. La population du pays, selon ces données, est donc officiellement de 207660929 habitants, en hausse de 0,77 \% par rapport à 2016 (206 081 432).

En examinant la période 2000-2017 (illustration 3), on constate que les communes dont la population a diminué (en bleu sur la carte) sont rares et petites ; pour la plupart elles 
ont grandi (jusqu'à $+50 \%$, en jaune) au cours de ces 17 ans et certaines ont même grandi très vite (de $+50 \%$ à près de $+300 \%$, en rouge). Ces dernières sont situées principalement sur les fronts pionniers amazoniens et à la périphérie des très grandes villes, Rio, Sao Paulo et Brasilia. La première a gagné dans cette période plus d'un million et demi d'habitants, la seconde un peu plus d'un demi-million et Brasília a atteint pour la première fois les 3 millions d'habitants, fait remarquable pour une ville fondée en 1960.

Illustration 3 - Évolution de la population entre 2000 et 2017

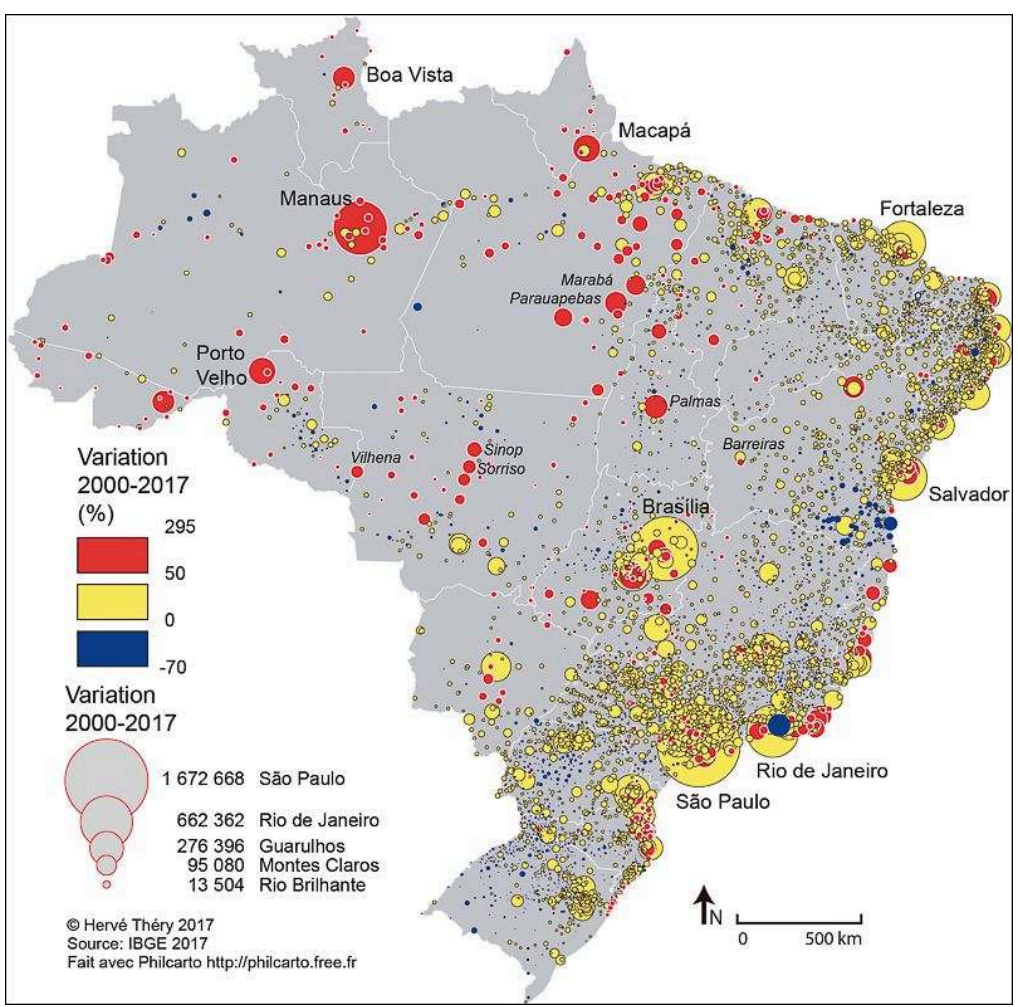

9 Cette croissance ne change pourtant pas fondamentalement la distribution, très inégale, de la population brésilienne sur le territoire. Une nette opposition persiste entre les régions littorales et intérieures, qui reflète aujourd'hui encore les effets du processus de colonisation et de peuplement du territoire par des migrants venus par la mer, volontairement (colons portugais et immigrants européens du XIX ${ }^{e}$ siècle) ou non (esclaves africains) : les premières sont très peuplées alors que les secondes ne sont que très faiblement occupées.

Les zones de concentration sont soulignées par la carte des densités démographiques (illustration 4), qui confirment le contraste littoral/intérieur. C'est dans les parties les plus proches de la mer des régions Nordeste, Sudeste et Sud que se trouvent les densités les plus élevées, au-dessus de 55 habitants par kilomètre carré, densités qui peuvent monter jusqu'à plus de 10000 habitants $/ \mathrm{km}^{2}$ dans les communes des capitales. Mais même dans les États les plus peuplés apparaissent des vides importants ; seuls São Paulo, le Paraná, Rio de Janeiro, le Sergipe, l'Alagoas, la Paraíba et le Ceará voient leur territoire occupé de façon continue.

11 À l'opposé, la majeure partie de l'Amazonie et de vastes surfaces du Centre-Ouest présentent des densités très basses, entre 0,1 et 2 habitants par kilomètre carré, dont 
ne se détachent que les capitales et quelques municípios (communes) aux effectifs un peu supérieurs. Dans ces régions de densité plus basse, la répartition de la population est étroitement corrélée avec les réseaux de transport, voies navigables en Amazonie, routes de desserte régionale et nationale ailleurs qui ont succédé aux chemins de fer construits naguère pour exporter les produits de l'intérieur (sucre, café et minerais).

Illustration 4 Densité démographique et transports

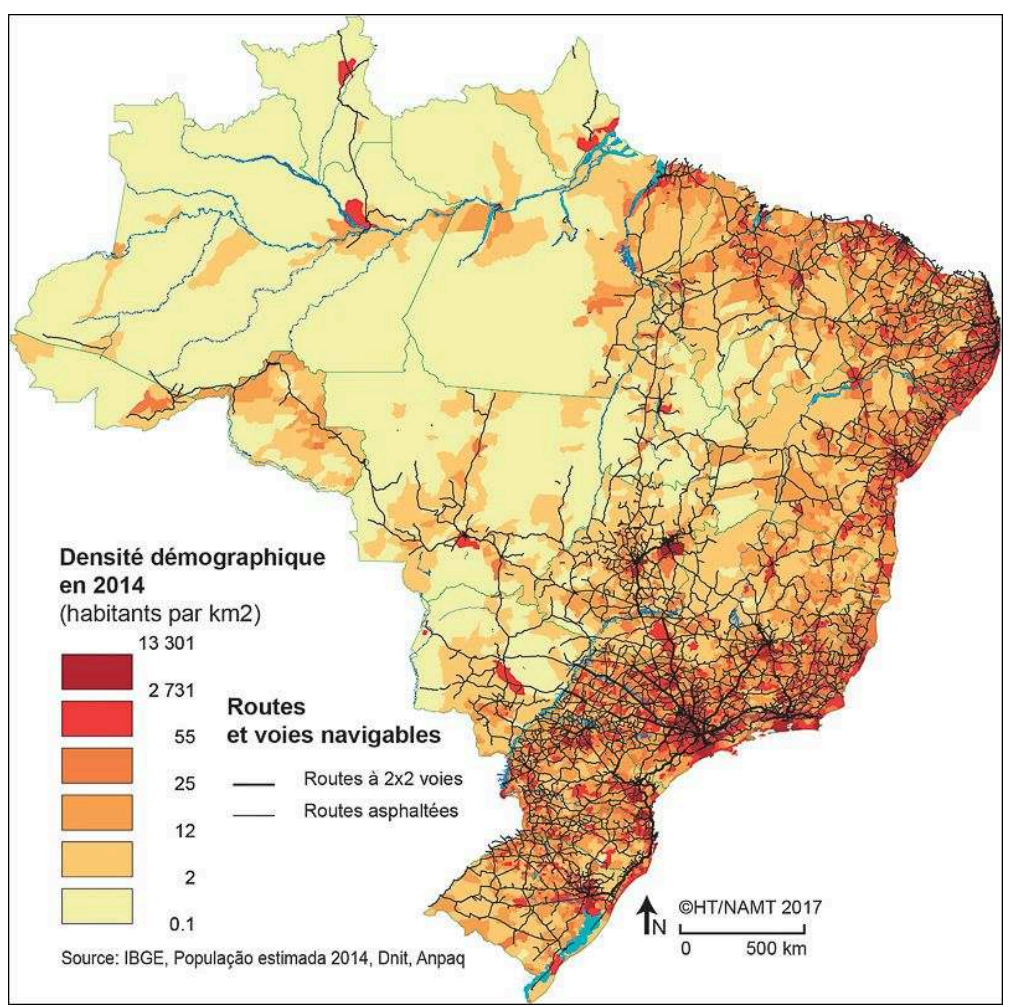

12 La lecture des articles publiés dans ce dossier montre que les auteurs des articles retenus contribuent à mettre à jour l'état des lieux du pays, sur bon nombre de points.

\section{Les articles}

\section{Espaces urbains et ruraux}

Dans leur article intitulé «La croissance urbaine du Brésil se concentre-t-elle dans les métropoles ? ", Cathy Chatel, François Moriconi-Ebrard et Maria Encarnação Beltrão Sposito répondent directement à la question posée dans l'appel à contributions, «la métropolisation en cours du réseau urbain se traduit-elle [...] par une perte de vitesse des petites villes et par la croissance concentrée dans seulement quelques grandes régions urbaines? ». Ils montrent que le rythme de la croissance des deux principales métropoles, São Paulo et Rio de Janeiro, ne cesse de baisser depuis un demi-siècle et qu'elle est désormais inférieure à la croissance nationale. Ils utilisent pour cela une méthodologie originale, qui consiste à reconstruire l'ensemble de la hiérarchie urbaine du pays à partir d'une définition strictement morphologique des agglomérations urbaines. Elle permet de vérifier qu'il existe un "attracteur de taille démographique » qui se situe actuellement autour de 500000 habitants. Elle met aussi en valeur le rôle 
des fonctions administratives et politiques territoriales, notamment celui de capitale d'État.

14 Cláudio Zanotelli donne ensuite un zoom à la fois sur un cas particulier et un point d'actualité en traitant de «L'espace du politique ou la politique de l'espace : l'éviction des quartiers populaires et les Jeux Olympiques de Rio de Janeiro». Il analyse le cas emblématique du quartier de Vila Autódromo, à Rio de Janeiro. En vue des Jeux Olympiques de 2016, de vastes travaux y ont été entrepris pour la mise en place de la ville olympique et une partie des habitants devait être relogée pour faire place aux projets d'aménagement prévus. Certains ont refusé de partir et Cláudio Zanotelli montre que ce conflit s'inscrit dans une histoire qui remonte à l'époque coloniale, où les classes populaires ont pendant longtemps été exclues du jeu politique et de la propriété foncière et immobilière.

15 À la question «Ces grands écarts existent-ils aussi à l'intérieur même des espaces ruraux? ", Cássio Arruda Boechat, Ana Carolina Gonçalves Leite et Carlos de Almeida Tolédo répondent par une "Archéologie de la question agraire au Brésil». Ils reconstituent la trajectoire historique des régions du Brésil dans une double conceptualisation des procédures d'expropriation, centrées soit sur l'esclavage (labor grabbing), soit sur l'appropriation privée de la terre (land grabbing), produisant ainsi une nouvelle approche théorique de la genèse de la question agraire au Brésil.

Viennent ensuite deux articles de synthèse sur des questions qui préoccupent toujours quand il est question du Brésil - et sur lesquels il était donc hors de question de faire l'impasse - la question de l'environnement et la question indienne.

\section{Environnement et question indienne}

17 L'article de Neli Aparecida de Mello-Théry, «Politiques environnementales brésiliennes: intentions et réalités" est une réflexion sur les politiques environnementales élaborées et mises en œuvre au Brésil ces quinze dernières années, et en particulier sur celles qui touchent à la conservation de la biodiversité et au changement climatique. Les contradictions, les avancées et les reculs de la politique environnementale des gouvernements de Luíz Inácio « Lula » da Silva et Dilma Rousseff y sont analysés: certains plans et programmes spécifiques (en particulier sur le déboisement de l'Amazonie) et la politique sur les changements climatiques ont permis des progrès, tandis que d'autres ont plutôt aggravé une situation déjà précaire, comme ce fut le cas de la Loi sur les Forêts (Código Florestal).

Protéger efficacement l'environnement suppose d'abord de le connaitre, et notamment d'en mesurer précisément le potentiel et les limitations. C'est ce que font Vincent Dubreuil, Karime Pechetti, Olivier Planchon et João Lima Santanna en revisitant une des méthodes classiques de la climatologie dans « Les types de climats annuels au Brésil : une application de la classification de Koppen de 1961 à 2015 ». Ils y proposent d'utiliser cette classification pour définir des Types de Climats Annuels (TCA) pour un ensemble de 208 stations, représentatives de la diversité climatique du Brésil et où les données moyennes mensuelles de températures et de précipitations disponibles pour la période 1961-2015 ont permis d'établir la fréquence des TCA. Le traitement statistique et cartographique de ces informations précise les limites des climats du Brésil en appliquant les concepts de noyaux forts, définis comme les régions où les TCA sont systématiquement ou majoritairement les mêmes, et d'aires de transition, régions où 
l'on trouve une grande diversité des TCA. Cette approche permet d'envisager une étude fine des transitions entre les types de climats tropicaux du nord et ceux du sud, subtropicaux et tempérés, et de discuter ces limites climatiques.

Dans son article «Le Brésil et ses Indiens, une réconciliation impossible? » FrançoisMichel Le Tourneau part du constat que, trente ans après la Constitution de 1988 qui, en ouvrant aux Indiens de larges droits fonciers, avait semblé aller vers la réparation du préjudice historique causé par la dépossession de leurs terres, la réconciliation attendue n'a pas eu lieu. Certes, ils disposent aujourd'hui d'un usufruit exclusif sur une portion considérable du territoire brésilien (environ 13,7 \%) mais la situation a évolué depuis une dizaine d'années à leur détriment. Après un rapide panorama historique de la question amérindienne depuis la colonisation portugaise jusqu'aux avancées de la fin du XX $\mathrm{X}^{\mathrm{e}}$ siècle, il montre comment la Constitution de 1988 a permis la consolidation d'un patrimoine foncier très important, alors que la population amérindienne est ultraminoritaire. L'auteur analyse l'inflexion qui s'est produite depuis plus de dix ans, dans un sens défavorable aux droits fonciers des Amérindiens, et montre combien la situation actuelle met à l'épreuve les protections dont ils bénéficient, avant de s'interroger sur les scénarios possibles pour les prochaines années.

Autre sujet qui s'articule avec les deux précédents, l'Amazonie est abordée dans ce dossier par trois articles. Leurs auteurs respectifs remettent en question des visions souvent sommaires et entachées par la double vision rose et noire de l'Eldorado et de l'Enfer vert, des mythes qui ne favorisent pas une vision objective de cette région du monde (sans même parler des mythiques guerrières qui lui ont donné son nom).

\section{Amazonie}

21 Abordant les «Effets des équipements hydroélectriques en Amazonie », Céline Broggio et Martine Droulers y voient «Un exemple de résilience territoriale». Leur article s'intéresse aux effets à moyen et long termes des barrages et centrales hydroélectriques implantés en Amazonie brésilienne depuis les années 1980 sur la réorganisation des territoires environnants. Après les perturbations importantes provoquées par leur installation, un processus de résilience s'engage sur ces territoires dans un contexte de post-front pionnier. Les auteures se demandent si l'analyse du cas du barrage de Tucurui (Pará) peut, avec trente ans de recul, apporter des éléments de réflexion sur la situation actuelle dans la région de Porto Velho (Rondônia) où deux grandes centrales hydroélectriques (Santo Antônio et Jirau) ont été installées à la fin des années 2000.

Allant à l'encontre des idées reçues, souvent catastrophistes, qui règnent sur l'Amazonie, François Laurent, Damien Arvor et les dix autres auteurs d'un article de synthèse de travaux récents sur la région voient un tournant environnemental en Amazonie causé par le découplage entre production et déforestation dont ils mesurent ampleur et limites. Ils partent du constat que l'Amazonie a connu depuis quelques années une baisse de la déforestation couplée à un développement économique. Cependant, ils observent, à l'échelle de l'Amazonie Légale et de la commune Paragominas (Pará), que si les grands et moyens producteurs ont évolué vers l'intensification et délaissé - en partie - les stratégies d'expansion, l'agriculture familiale reste à l'écart de ce découplage. 
La progression de ces puissant front pionniers des bûcherons, des éleveurs de bovins, et des producteurs de soja, ne doit pourtant pas faire oublier la vieille Amazonie des fleuves, qui subsiste malgré l'avancée de l'Amazonie des routes qui s'y est superposée et a imposé ses propres réseaux depuis la construction de la Transamazonienne et des routes Belém-Brasília, Cuiabá-Santarém et Cuiabá-Porto Velho, dans les années 1970. Dans «Ribeirinho Food Regimes, Socioeconomic Inclusion and Unsustainable Development of the Amazonian Floodplain", Tatiana Schor et Gustavo S. Azenha examinent les changements dans l'acquisition des aliments et les habitudes alimentaires, ce qui les amène à explorer de manière critique le développement durable de l'Amazonie. Ce sont en effet de bons indicateurs des changements dans les perceptions et les usages de la nature en Amazonie, ils fournissent un moyen d'examiner l'écart entre les mythes de la durabilité et la réalité de l'urbanisation rapide et de l'évolution des moyens de subsistance dans l'Amazonie contemporaine. Les auteurs avancent qu'une écologie politique des régimes alimentaires est un moyen privilégié d'éclairer les contradictions entre les trajectoires actuelles du développement et les disparités socio-environnementales qu'elles engendrent, et peut contribuer à la formulation de plus efficaces politiques de développement durable et d'inclusion sociale.

24 Enfin, deux articles abordent la question régionale, l'un dans une perspective théorique et l'autre sur un cas particulier, celui de Brasilia.

\section{La question régionale}

25 Simone Affonso da Silva s'attache à "La problématique régionale brésilienne " en l'abordant par l'analyse de quelques controverses et leurs applications. Son article reprend les lectures - divergentes - qu'ont faites de l'origine des inégalités régionales au Brésil quatre intellectuels qui ont étudié les problèmes régionaux dans le pays: Celso Furtado, Wilson Cano, Francisco de Oliveira et Ruy Moreira. Puis il traite des principales politiques menées par le gouvernement fédéral brésilien depuis le milieu du $\mathrm{XX}^{\mathrm{e}}$ siècle afin d'intervenir politiquement, économiquement et socialement dans la dynamique socio-spatiale qui est au cœur de la question régionale. S'y distinguent les pôles de développement, les centres nationaux d'intégration et de développement et la politique nationale pour le développement régional. Toutes ces politiques sont marquées par des idéologies et des projets politiques sous-jacents, liés aux objectifs des acteurs au pouvoir ou qu'ils se sont appropriés. Cela doit être compris pour comprendre le rôle des inégalités régionales dans le panorama du développement socioéconomique brésilien.

Pour cas très spécifique de Brasília, Renata Callaça Gadioli dos Santos, Luiz Fernando de Macedo Bessa et Magda de Lima Lúcio étudient sa place dans les politiques nationales dans «The Brazilian National Policy for Regional Development and the RIDE-DF Management vs the governance of Brasilia's Metropolitan Area». Malgré la proposition de mise à jour de la politique nationale brésilienne pour le développement régional (PNDR), la gouvernance du territoire métropolitain de Brasilia, (qui est identifié comme un domaine prioritaire de planification et de gestion) continue à faire l'objet de conflits et maintient l'inégalité dans ses zones périphériques. Pour contribuer au débat sur la portée possible du PNDR dans la gestion de cette ville importante et complexe, les 
auteurs proposent une analyse théorique et méthodologique du modèle de gouvernance urbaine et de la relation entre PNDR I et II avec la RIDE-DF et Entorno.

Dans ce dossier, la collaboration de «brésilianistes » français et d'auteurs brésiliens a donc permis de d'alimenter un dossier fourni d'articles sur quelques-uns des sujets principaux de l'actualité du Brésil, que ce soient des sujets habituellement abordés ou d'autres plus originaux mais qui pèsent au moins autant dans l'évolution du pays. Boa leitura...

\section{NOTES}

1. De son nom complet Antônio Carlos Brasileiro de Almeida Jobim, " père " de la bossa nova et compositeur avec Vinicius de Moraes de A garota de Ipannema/The girl from Ipanema, la deuxième chanson pop la plus enregistrée dans l'histoire, après Yesterday par les Beatles. L'aéroport international de Rio de Janeiro porte aujourd'hui son nom.

2. «Brésil 2016, l'écume et les courants profonds", EchoGéo, Sur le Vif, URL: http:// echogeo.revues.org/14645; DOI : 10.4000/echogeo.14645

3. Inde: le grand écart spatial, https://echogeo.revues.org/14220, notamment l'introduction de Frédéric Landy, EchoGéo 32 | 2015, http://echogeo.revues.org/14279

4. http://www1.folha.uol.com.br/cotidiano/2017/08/1914234-brasil-atinge-a-marca-de-207milhoes-de-habitantes-afirma-ibge.shtml

\section{AUTHOR}

\section{HERVÉ THÉRY}

Hervé Théry, herve.thery@usp.br, est Directeur de recherche émérite au CNRS-Creda, Professeur à l'Universidade de São Paulo- USP (PPGH/FFLCH et GPP/EACH). 